\title{
Comparison of clinical characteristics and outcomes between respiratory syncytial virus and influenza-related pneumonia in China from 2013 to 2019
}

\author{
Liang Chen ${ }^{1}$ (D) Xiudi $\mathrm{Han}^{2} \cdot$ YanLi $\mathrm{Li}^{3} \cdot$ Chunxiao Zhang $^{4} \cdot$ Xiqian Xing $^{5}$ \\ Received: 19 October 2020 / Accepted: 28 February 2021 / Published online: 6 March 2021 \\ (C) The Author(s), under exclusive licence to Springer-Verlag GmbH Germany, part of Springer Nature 2021
}

\begin{abstract}
This study aims to compare clinical characteristics and severity between adults with respiratory syncytial virus (RSV-p) and influenza-related pneumonia (Flu-p). A total of 127 patients with RSV-p, 693 patients with influenza A-related pneumonia (FluA-p), and 386 patients with influenza B-related pneumonia (FluB-p) were retrospectively reviewed from 2013 through 2019 in five teaching hospitals in China. A multivariate logistic regression model indicated that age $\geq 50$ years, cerebrovascular disease, chronic kidney disease, solid malignant tumor, nasal congestion, myalgia, sputum production, respiratory rates $\geq 30$ beats $/ \mathrm{min}$, lymphocytes $<0.8 \times 10^{9} / \mathrm{L}$, and blood albumin $<35 \mathrm{~g} / \mathrm{L}$ were predictors that differentiated RSV-p from Flu-p. After adjusting for confounders, a multivariate logistic regression analysis confirmed that, relative to RSV-p, FluA-p (OR 2.313, 95\% CI 1.377-3.885, $p=0.002$ ) incurred an increased risk for severe outcomes, including invasive ventilation, ICU admission, and 30-day mortality; FluB-p $(O R$ 1.630, 95\% CI 0.958-2.741, $p=0.071)$ was not associated with increased risk. Some clinical variables were useful for discriminating RSV-p from Flu-p. The severity of RSV-p was less than that of FluA-p, but was comparable to FluB-p.
\end{abstract}

Keywords Respiratory syncytial virus $\cdot$ Influenza virus $\cdot$ Pneumonia $\cdot$ Clinical characteristic $\cdot$ Outcome

\section{Introduction}

Influenza is a common viral contagious disease, and epidemics or pandemics have occurred all over the world [1]. Despite economic and medical advances, influenza causes

Liang Chen

chenliang1995@sina.com

1 Department of Infectious Diseases, Beijing Jishuitan Hospital, 4th Medical College of Peking University, Beijing, China

2 Department of Pulmonary and Critical Care Medicine, Qingdao Municipal Hospital, Qingdao City, Shandong Province, China

3 Department of Infectious Diseases and Clinical Microbiology, Beijing Chao-Yang Hospital, Capital Medical University, Beijing, China

4 Department of Pulmonary and Critical Care Medicine, Beijing Huimin Hospital, Beijing, China

5 Department of Pulmonary and Critical Care Medicine, The Second People's Hospital of Yunnan Province, Kunming City, Yunnan Province, China considerable morbidity and mortality [2, 3]. It is estimated that during an annual seasonal epidemic, $10-20 \%$ of the global population experienced symptomatic influenza, which included 3-5 million cases of severe illness and 290,000-650,000 deaths from influenza-related respiratory complications [4].

Human respiratory syncytial virus (RSV) is one of the most common viruses to infect children worldwide [5] and is increasingly recognized as an important cause of respiratory infections in adults, particularly the elderly and those with underlying chronic conditions [6,7]. It has been estimated that each year RSV infects 3-7\% of healthy elderly patients and 410\% of high-risk adults [8]. From 1997 to 2009, RSV contributed to approximately 17,000 annual cardiorespiratory deaths in China [9]. A study conducted in a Wisconsin community estimated the overall seasonal incidences of medically attended RSV illness to be $154 / 10,000$ in individuals aged $\geq$ 50 years and 199/10,000 among persons aged $\geq 70$ years [10]. It is believed that both the incidence of hospitalization and the mortality rate of RSV infection are comparable to, and perhaps even higher than, non-pandemic influenza virus [11]. 
Both influenza virus and RSV are important causes of pneumonia, and these pathogens are implicated in $5-15 \%$ of community-acquired pneumonia cases in adults $[12,13]$. Meanwhile, nearly $40-50 \%$ of severe influenza and RSV cases that result in hospitalizations and/or deaths present with pneumonia at some point, including primary viral pneumonia and secondary bacterial pneumonia $[14,15]$. Unlike influenza, no licensed RSV vaccine is available, and the only approved specific therapy, palivizumab (anti-RSV antibody), is of limited use with infants [16]. Early differential diagnosis between the two respiratory viruses is critical for arranging prompt treatment and for making rational clinical decisions. One difficulty, however, is that influenza and RSV often present similar symptoms and they occur during the same season [17-19]. The overlapping seasonality of the two viruses makes them difficult to distinguish. Based on our review of the relevant research, it appears that no large-scale study has investigated the differences in the clinical characteristics of adults hospitalized with RSV-p versus Flu-p.

To address this omission from the literature, we conducted a multicenter cohort study with two principal aims: (i) to clinically differentiate RSV-p from Flu-p and (ii) to evaluate the impact of virus type on illness severity and pneumonia-related outcomes.

\section{Methods}

\section{Study design and participants}

The medical records of hospitalized patients testing positive for influenza and RSV RNA at the Microbiology Labs of five teaching hospitals in China from January 1, 2013 through May 31, 2019 were reviewed (details of the participating centers can be found in Supplementary Material 1). Patients with laboratory-confirmed Flu-p and RSV-p were included. Exclusion criteria were as follows: (i) being less than 18 years of age, (ii) not classified as community-onset pneumonia (i.e., pneumonia onset $\geq 48 \mathrm{~h}$ post-admission and hospitalized within the last 28 days [20]), (iii) coinfection with other respiratory viruses, and (iv) immunocompromised status. The last point is important because the clinical characteristics and outcomes of immunocompromised patients with influenza and RSV might be different from similarly infected immunocompetent hosts $[21,22]$.

\section{Disease and treatment definitions}

Patients with Flu-p or RSV-p were defined as patients positive for influenza virus or RSV by reverse transcription polymerase chain reaction (RT-PCR) performed on respiratory specimens (i.e., nasal/nasopharyngeal swabs, sputum, bronchial aspirates, or branchoalveolar lavage fluid) and who presented with respiratory symptoms along with newly emerging pulmonary infiltrates on chest radiographs. Early neuraminidase inhibitor (NAI) therapy was defined as any NAI (oseltamivir, zanamivir, and peramivir) administered within $48 \mathrm{~h}$ of illness onset [23]. Treatment consisted of systemic corticosteroid use, defined as at least one dose of any systemic corticosteroid administered during hospitalization. Community-acquired respiratory coinfected pathogens were defined as any pathogen identified within the first $48 \mathrm{~h}$ after admission using standard microbiological procedures (the microbiological criteria for identifying coinfection are presented in Supplementary Material 2) [24]. Severe outcomes were defined as any of the following: invasive ventilation, intensive care unit (ICU) admission, or death within 30 days after admission.

\section{Data collection}

All data were retrospectively collected and included demographic information, chronic underlying conditions (see Supplementary Material 3 for definitions of conditions/ comorbidities), clinical symptoms, vital signs, laboratory and radiological findings at admission, community-acquired respiratory coinfections, and clinical management and outcomes (e.g., administration of systemic corticosteroids and vasopressor agents, invasive and non-invasive mechanical ventilation, complications during hospitalization, admittance to the ICU, length of hospital stay, cost of hospitalization, and 30-day mortality). Those patients with hospital stays $<30$ days were followed up via phone call to determine survival status.

\section{Statistical analysis}

Distributional normality assumptions were examined using Kolmogorov-Smirnov tests. Variables evidencing normal distributions are presented as the mean $\pm \mathrm{SD}$. Those variables with non-normal distributions are expressed as the median (interquartile range). Categorical variables were analyzed using either the chi-squared test or Fisher's exact test. Continuous variables were analyzed using Student's $t$-test or the Mann-Whitney $U$ test. For all analyses, two-tailed probability values $\leq 0.05$ were considered statistically significant.

We compared the demographic and baseline clinical features between patients diagnosed with RSV-p versus those infected with Flu-p. Variables with $p$-values $<0.1$ in the univariate and bivariate analyses were then entered into a multivariate logistic regression model to identify the predictors of RSV-p.

In an effort to account for potential confounders, the multivariate logistic regression model was controlled for age, sex, duration from illness onset to admission, comorbidities, pregnancy, obesity, smoking history, early NAI therapy, systemic corticosteroid use, and coinfection with other pathogens. Because these factors have been shown to correlate with 
clinical outcomes in patients with influenza and other respiratory virus infections [23], we adjusted for these variables when conducting our multivariate analysis.

Additional analyses compared the baseline characteristics of RSV-p patients with and without severe outcomes. Those variables with $p$-values $<0.1$ were also entered into the multivariate logistic regression analysis. All statistical analyses were performed using SPSS Statistics version 22.0.

\section{Results}

\section{Screening process}

We screened 3375 patients who were RSV or influenza RNA positive. A total of 127 laboratory-confirmed RSV-p patients and 1079 Flu-p patients (including 693 FluA-p patients and 386 FluB-p patients) were included in the study (Fig. 1). Of the subset of FluA-p patients, 38.1\% (264/693) were infected with A (H1N1) pmd09, $11.0 \%$ (76/693) were infected with A (H3N2), and 50.9\% (353/693) were infected with an unclassified subtype.

\section{Monthly distribution of patients with RSV-p and Flu-p}

The monthly distribution of patients with RSV-p and Flu-p is presented in Fig. 2. Generally, the cases of RSV-p and Flu-p showed a similar seasonal pattern, with both infections occurring from October through May. The peak of RSV-p was from November through January. In contrast, the peaks of FluA-p and FluB-p were from December through February and January through March, respectively.

\section{Overview of patients with Flu-p}

In total, $74.4 \%$ (803/1079) of Flu-p patients were age 50 years and older, and males accounted for $54.1 \%$ (584/1079) of cases. In addition, 42.4\% (457/1079) of Flu-p patients had at least one underlying disease, with the three most prevalent being cardiovascular disease $(24.0 \%, 259 / 1079)$, diabetes mellitus $(11.8 \%, 127 / 1079)$, and cerebrovascular disease $(9.0 \%, 97 / 1079)$. Other findings were that 29.0\% (313/1079) of Flu-p patients had a history of smoking, $98.2 \%$ (1060/1079) presented with cough, $79.1 \%$ (854/1079) had sputum production, and $75.4 \%(814 / 1079)$ had a fever. $\mathrm{PO}_{2} /$ $\mathrm{FiO}_{2}<250 \mathrm{mmHg}$ and multilobar infiltrates on chest radiology were evident in $30.2 \%$ (310/1025) and $73.6 \%$ (794/1079) of Flu-p patients, respectively (Table 1). The chest radiology of pneumonia related to influenza and RSV could be seen in Supplementary Fig. 1.

Supplementary Material 4 shows that $34.0 \%$ (367/1079) of Flu-p patients were coinfected with other communityacquired pathogens. The most common coinfected pathogens were Klebsiella pneumoniae (31.6\%, 116/367), Streptococcus
Fig. 1 Screening algorithm of patients hospitalized with RSV-p and Flu-p. The medical records of 3375 patients with RSV or influenza RNA positive were reviewed. A total of 127 laboratory-confirmed RSV-p patients and 1079 Flu-p patients (including 693 FluA-p patients and 386 FluB-p patients) were included

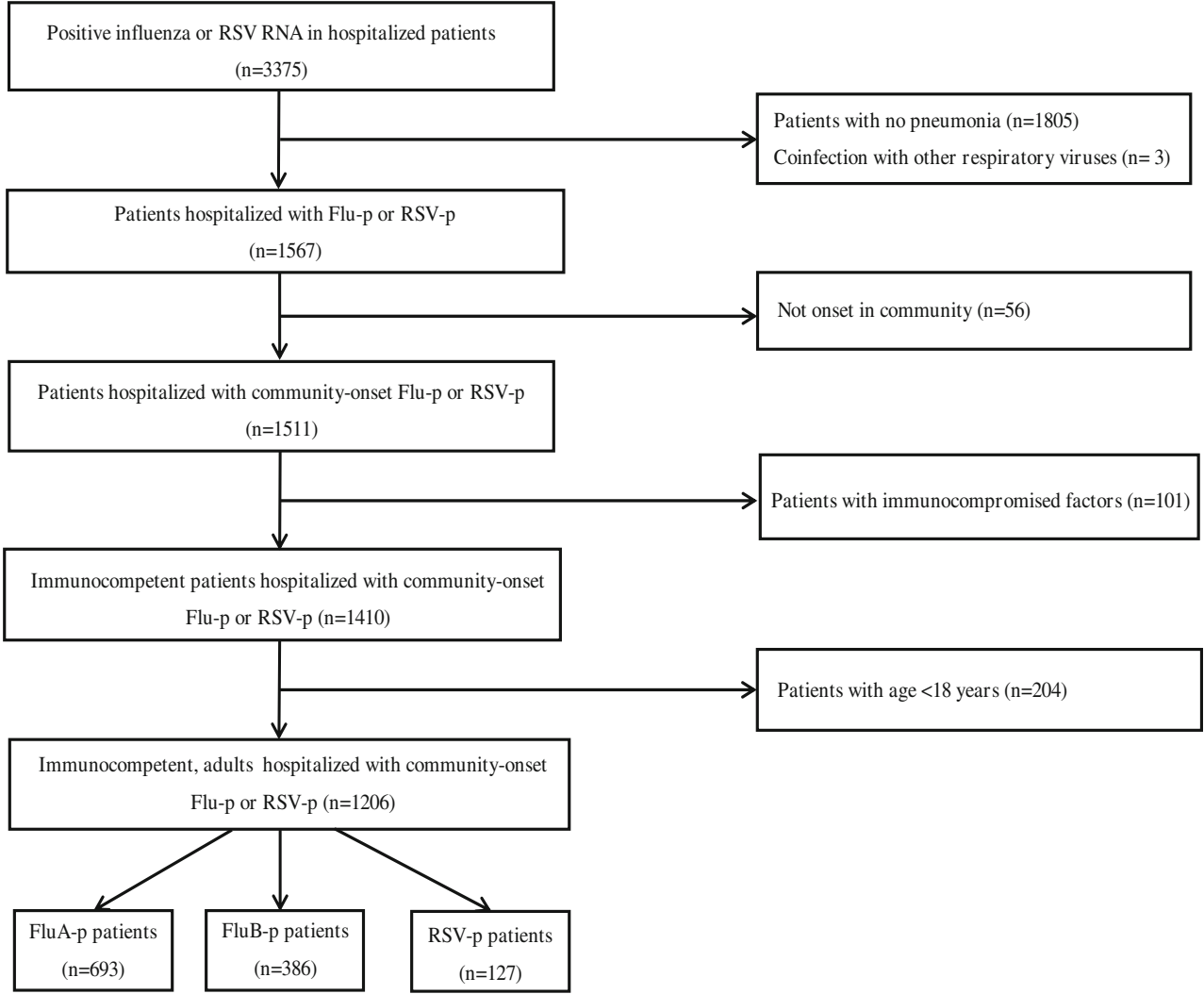


Fig. 2 Distribution of patients with RSV-p and Flu-p by months. RSV-p and Flu-p had similar seasonality, both covered from October to May

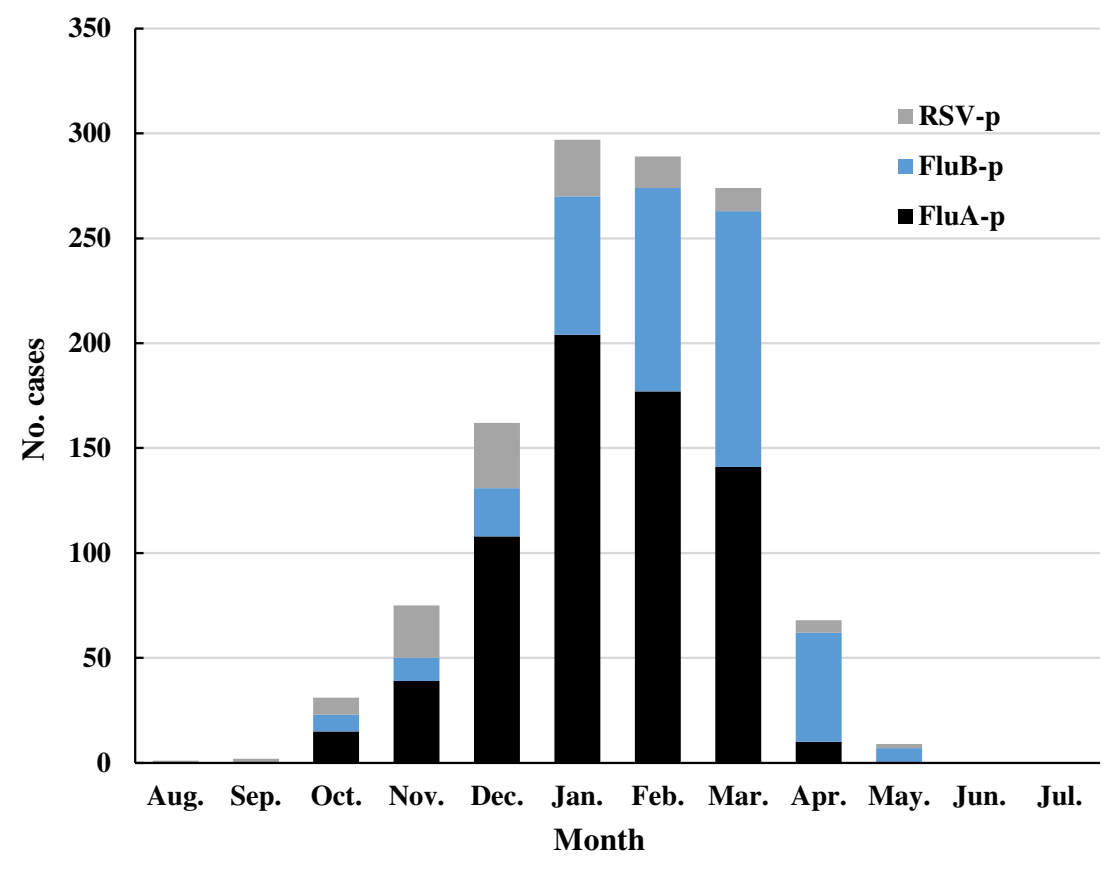

pneumoniae (29.7\%, 109/367), and Staphylococcus aureus (19.3\%, 71/1079), respectively.

Although only $34.0 \%$ of Flu-p patients were coinfected with other community-acquired pathogens, all Flu-p patients were administrated with antibiotics after admission, and NAI during disease course, with early NAI, was administrated to $35.7 \%(385 / 1079)$ of patients. In total, $24.3 \%(262 / 1079)$ of Flu-p patients received systemic corticosteroids during hospitalization. Regarding adverse outcomes, 23.1\% (249/1079) developed respiratory failure, $24.6 \%$ (265/1079) experienced heart failure, and $8.2 \%$ (89/1079) developed septic shock, respectively. In total, $17.9 \%$ (193/1079) of Flu-p patients received invasive ventilation and $22.4 \%$ (242/1079) were admitted to the ICU. The 30-day mortality rate for Flu-p patients was $19.3 \%$ (208/1079) (Table 2).

\section{Overview of patients with RSV-p}

In total, $59.1 \%(75 / 127)$ of RSV-p patients were male, and $96.9 \%(123 / 127)$ were age 50 years or older. Underlying medical conditions affected $65.4 \%$ (83/127) of RSV-p patients, and the top three conditions were cardiovascular disease $(34.6 \%, 44 / 127)$, chronic pulmonary disease $(23.6 \%, 30 /$ 127), and cerebrovascular disease $(15.7 \%, 20 / 127)$. Cough $(96.1 \%, 122 / 127)$ was the most common symptom, with sputum production $(11.0 \%, 14 / 127)$ occurring significantly less often. Dyspnea and fever were present in 71.7\% (91/127) and $55.9 \%(71 / 127)$ of RSV-p patients, respectively (Table 1).

Coinfections were isolated in $30.7 \%$ (39/127) of RSV-p patients. The top three coinfected pathogens were K. pneumoniae (48.7\%, 19/39), S. aureus (20.5\%, 8/39), and other Streptococcus spp. (7.7\%, 3/39), respectively (see Supplementary Material 4).

All RSV-p patients received antibiotics, and 7.9\% (10/127) were administered systemic corticosteroids during hospitalization. Noninvasive and invasive ventilation were performed in $25.2 \%(32 / 127)$ and $11.0 \%$ (14/127) of RSV-p patients, respectively. Heart failure $(33.1 \%, 42 / 127)$ was the most frequent complication, followed by respiratory failure $(29.1 \%, 37 / 127)$ and acute renal failure $(6.3 \%, 8 / 127)$. In total, $11.0 \%(14 / 127)$ of RSV-p patients were admitted to the ICU, and the 30-day mortality rate was $14.2 \%(18 / 127)$ (Table 2$)$.

\section{Predictors of RSV-p}

Compared with Flu-p, bivariate analyses indicated that RSV-p was associated with age $\geq 50$ years, cardiovascular disease, cerebrovascular disease, chronic pulmonary disease, chronic kidney disease, solid malignant tumor, fever, myalgia, sputum production, respiratory rates $\geq 30$ beats/min, lymphocytes < $0.8 \times 10^{9} / \mathrm{L}$, and blood albumin $<35 \mathrm{~g} / \mathrm{L}$ (Table 1 ).

A multivariate logistic regression model indicated that age $\geq$ 50 years (odds ratio $(O R) 11.207,95 \%$ confidence interval (CI) 3.266-38.456, $p<0.001)$, cerebrovascular disease (OR 4.189, 95\% CI 1.473-11.918, $p=0.007)$, chronic kidney disease $(O R$ 8.934, 95\% CI 2.114-37.760, $p=0.003$ ), solid malignant tumor (OR 3.407, 95\% CI 1.102-10.533, $p=0.033$ ), nasal congestion (OR 4.088, 95\% CI 1.857-8.999, $p<0.001)$, respiratory rates $\geq$ 30 beats $/ \min (O R 2.612,95 \% C I 1.105-6.173, p=0.029)$, and blood albumin $<35 \mathrm{~g} / \mathrm{L}$ (OR 6.454, 95\% CI 2.842-14.661, $p<$ 0.001 ) were all positively associated with RSV-p, while myalgia (OR $0.126,95 \%$ CI $0.056-0.285, p<0.001)$, sputum production (OR 0.006, 95\% CI 0.003-0.014, $p<0.001)$, and lymphocytes $<$ 
Table 1 Comparison of demographical and clinical features between patients with Flu-p and RSV-p

\begin{tabular}{|c|c|c|c|c|c|}
\hline Variable & $\begin{array}{l}\text { Flu-p } \\
(n=1079)\end{array}$ & $\begin{array}{l}\text { FluA-p } \\
(n=693)\end{array}$ & $\begin{array}{l}\text { FluB-p } \\
(n=386)\end{array}$ & $\begin{array}{l}\text { RSV-p } \\
(n=127)\end{array}$ & $P$ value $^{\dagger}$ \\
\hline Male $(n, \%)$ & $584(54.1)$ & $461(66.5)$ & $123(31.9)$ & $75(59.1)$ & 0.291 \\
\hline Age $\geq 50$ years $(n, \%){ }^{\#}$ & $803(74.4)$ & $463(66.8)$ & $340(88.1)$ & $123(96.9)$ & $<0.001$ \\
\hline Duration from illness onset to admission (days, median, IQR) & $3.0(2.0-4.0)$ & $3.0(2.0-4.0)$ & $3.0(2.0-4.3)$ & $3.5(2.0-5.0)$ & 0.628 \\
\hline Chronic medical condition $(n, \%)$ & $457(42.4)$ & $262(37.8)$ & $195(50.5)$ & $83(65.4)$ & $<0.001$ \\
\hline Cardiovascular disease $^{\#}$ & $259(24.0)$ & $136(19.6)$ & $123(31.9)$ & $44(34.6)$ & 0.009 \\
\hline Cerebrovascular disease $^{\#}$ & $97(9.0)$ & $72(10.4)$ & $25(6.5)$ & $20(15.7)$ & 0.015 \\
\hline Diabetes mellitus & $127(11.8)$ & $92(13.3)$ & $35(9.1)$ & $19(15.0)$ & 0.297 \\
\hline $\mathrm{COPD}^{\#}$ & $91(8.4)$ & $40(5.8)$ & $51(13.2)$ & $30(23.6)$ & $<0.001$ \\
\hline Asthma & $33(3.1)$ & $19(2.7)$ & $14(3.6)$ & $5(3.9)$ & 0.789 \\
\hline $\mathrm{CKD}^{\#}$ & $30(2.8)$ & $16(2.3)$ & $14(3.6)$ & $14(11.0)$ & $<0.001$ \\
\hline Solid malignant tumor ${ }^{\#}$ & $24(2.2)$ & $16(2.3)$ & $8(2.1)$ & $16(12.6)$ & $<0.001$ \\
\hline Obesity & $76(7.0)$ & $48(6.9)$ & $28(7.3)$ & $8(6.3)$ & 0.755 \\
\hline Pregnancy & $8(0.7)$ & $8(1.2)$ & $0(0.0)$ & $0(0.0)$ & 1.000 \\
\hline Smoking history & $313(29.0)$ & $243(35.1)$ & $70(18.1)$ & $36(28.3)$ & 0.876 \\
\hline \multicolumn{6}{|l|}{ Baseline clinical features $(n, \%)$} \\
\hline Fever $\geq 38^{\circ} \mathrm{C}^{\#}$ & $814(75.4)$ & $661(95.4)$ & $153(39.6)$ & $71(55.9)$ & $<0.001$ \\
\hline Sore throat & $202(18.7)$ & $163(23.5)$ & $39(10.1)$ & $23(18.1)$ & 0.867 \\
\hline Runny nose $\mathrm{e}^{\#}$ & $234(21.7)$ & $155(22.4)$ & $79(20.5)$ & $36(28.3)$ & 0.089 \\
\hline Nasal congestion ${ }^{\#}$ & $194(18.0)$ & $155(22.4)$ & $39(10.1)$ & $38(29.9)$ & 0.002 \\
\hline Myalgia\# & $376(34.8)$ & $208(30.0)$ & $168(43.5)$ & $19(15.0)$ & $<0.001$ \\
\hline Cough & $1060(98.2)$ & $679(98.0)$ & $381(98.7)$ & $122(96.1)$ & 0.185 \\
\hline Sputum production ${ }^{\#}$ & $854(79.1)$ & $539(77.8)$ & $315(81.6)$ & $14(11.0)$ & $<0.001$ \\
\hline Dyspnea $^{\#}$ & $690(63.9)$ & $412(59.5)$ & $278(72.0)$ & $91(71.7)$ & 0.086 \\
\hline Thoracodynia & $182(16.9)$ & $112(16.2)$ & $70(18.1)$ & $26(20.5)$ & 0.309 \\
\hline Confusion & $150(13.9)$ & $32(4.6)$ & $118(30.6)$ & $16(12.5)$ & 0.687 \\
\hline Respiratory rates $\geq 30$ beats $/ \mathrm{min}^{\#}$ & $146(13.5)$ & $121(17.5)$ & $25(6.5)$ & $26(20.5)$ & 0.034 \\
\hline $\mathrm{SBP}<90 \mathrm{mmHg}$ & $15(1.4)$ & $8(1.2)$ & $7(1.8)$ & $2(1.6)$ & 1.000 \\
\hline Leukocytes $>10 \times 10^{9} / \mathrm{L}$ & $283(26.2)$ & $118(17.0)$ & $165(42.7)$ & $28(22.0)$ & 0.308 \\
\hline Lymphocytes $<0.8 \times 10^{9} / \mathrm{L}^{\#}$ & $480 / 1063(45.2)$ & $299 / 677(44.2)$ & $181(46.9)$ & $14(11.0)$ & $<0.001$ \\
\hline $\mathrm{HB}<100 \mathrm{~g} / \mathrm{L}$ & $240(22.2)$ & $69(10.0)$ & $171(44.3)$ & $28(22.0)$ & 0.960 \\
\hline ALB $<35 \mathrm{~g} / \mathrm{L}^{\#}$ & $187 / 1025(17.3)$ & $58 / 639(9.1)$ & $129(33.4)$ & $34(26.8)$ & 0.021 \\
\hline $\mathrm{BUN}>7 \mathrm{mmol} / \mathrm{L}$ & 446/1071 (41.6) & $183 / 685(26.7)$ & $263(68.1)$ & $49(38.6)$ & 0.508 \\
\hline $\mathrm{PaO}_{2} / \mathrm{FiO}_{2}<250 \mathrm{mmHg}$ & $310 / 1025(30.2)$ & $172 / 639(26.9)$ & $138(35.8)$ & $35(27.6)$ & 0.533 \\
\hline Multilobar infiltrate & $794(73.6)$ & $546(78.8)$ & $248(64.2)$ & $87(68.5)$ & 0.277 \\
\hline Coinfections $(n, \%)$ & $367(34.0)$ & $265(38.2)$ & $102(26.4)$ & $39(30.7)$ & 0.456 \\
\hline
\end{tabular}

Flu- $p$ influenza-related pneumonia, FluA- $p$ influenza A-related pneumonia, FluB- $p$ influenza B-related pneumonia, $R S V-p$ respiratory syncytial virusrelated pneumonia, $I Q R$ interquartile range, $C O P D$ chronic obstructive pulmonary disease, $C K D$ chronic kidney disease, $S B P$ systolic blood pressure, $\mathrm{HB}$ hemoglobin, $\mathrm{ALB}$ albumin, $\mathrm{BUN}$ blood urea nitrogen, $\mathrm{PaO}_{2} / \mathrm{FiO}_{2}$ arterial pressure of oxygen/fraction of inspiration oxygen. ${ }^{\#}$ Variables cited in the table above were the candidates that were entered into the multivariate logistic regression model. ${ }^{\dagger}$ Comparisons were made between patients with Flu-p and RSV-p. The bolded values are $p$-values $<0.05$, which represented significant differences between patients with Flu-p and RSV-p.

$0.8 \times 10^{9} / \mathrm{L}($ OR $0.145,95 \%$ CI $0.053-0.398, p<0.001)$ were negatively related to RSV-p (Fig. 3).

\section{Impact of virus type on severity of outcomes}

The effects of virus type on the severity of outcomes are presented in Table 3. Univariate logistic regression analyses indicated that, relative to RSV-p, FluA-p was associated with an increased risk for severe outcomes (OR 1.931, $95 \% \mathrm{CI}$ $1.241-3.005, p=0.004)$, including invasive ventilation $(O R$ 2.384, 95\% CI 1.331-4.271, $p=0.003$ ) and ICU admission (OR 2.748, 95\% CI 1.537-4.913, $p=0.001$ ). In contrast, the 30-day mortality rate was not significant for FluA-p (OR 1.479, 95\% CI 0.868-2.519, $p=0.150)$. Regarding FluB-p 
Table 2 Comparison of clinical management and outcomes between patients with Flu-p and RSV-p

\begin{tabular}{|c|c|c|c|c|c|}
\hline Variable & $\begin{array}{l}\text { Flu-p } \\
(n=1079)\end{array}$ & $\begin{array}{l}\text { FluA-p } \\
(n=693)\end{array}$ & $\begin{array}{l}\text { FluB-p } \\
(n=386)\end{array}$ & $\begin{array}{l}\text { RSV-p } \\
(n=127)\end{array}$ & $\begin{array}{l}P- \\
\text { value }^{\dagger}\end{array}$ \\
\hline Early NAI therapy & $385(35.7)$ & $232(33.5)$ & $153(39.6)$ & $0(0.0)$ & $\begin{array}{l}< \\
0.0- \\
01\end{array}$ \\
\hline $\begin{array}{l}\text { Systemic corticosteroid use } \\
\quad(n, \%)\end{array}$ & $262(24.3)$ & $132(19.0)$ & $130(33.7)$ & $10(7.9)$ & $\begin{array}{l}< \\
0.0- \\
01\end{array}$ \\
\hline $\begin{array}{l}\text { Noninvasive ventilation }(n \text {, } \\
\%)\end{array}$ & $279(25.9)$ & $159(22.9)$ & $120(31.1)$ & $32(25.2)$ & 0.872 \\
\hline Invasive ventilation $(n, \%)$ & $193(17.9)$ & $158(22.8)$ & $35(9.1)$ & $14(11.0)$ & 0.052 \\
\hline Vasopressor use $(n, \%)$ & $40(3.7)$ & $27(3.9)$ & $13(3.4)$ & $6(4.7)$ & 0.748 \\
\hline \multicolumn{6}{|l|}{ Complications $(n, \%)$} \\
\hline Respiratory failure & $249(23.1)$ & $167(24.1)$ & $82(21.2)$ & $37(29.1)$ & 0.129 \\
\hline Heart failure & $265(24.6)$ & $147(21.2)$ & $118(30.6)$ & $42(33.1)$ & $\begin{array}{l}<0 . \\
01 \\
01\end{array}$ \\
\hline Septic shock & $89(8.2)$ & $53(4.9)$ & $36(5.2)$ & $7(5.5)$ & 0.281 \\
\hline Acute renal failure & $66(6.1)$ & $39(5.6)$ & $27(7.0)$ & $8(6.3)$ & 0.935 \\
\hline Gastrointestinal bleeding & $48(4.4)$ & $40(5.8)$ & $8(2.1)$ & $3(2.4)$ & 0.269 \\
\hline $\begin{array}{l}\text { Admittance to the ICU ( } n, \\
\%)\end{array}$ & $242(22.4)$ & $176(25.4)$ & $66(17.1)$ & $14(11.0)$ & 0.003 \\
\hline $\begin{array}{l}\text { Length of stay in hospital } \\
\text { (days, median, IQR) }\end{array}$ & $\begin{array}{l}10.0 \\
\quad(8.0-14.0)\end{array}$ & $\begin{array}{l}12.0 \\
\quad(7.0-14.5)\end{array}$ & $\begin{array}{l}10.0 \\
\quad(8.0-17.0)\end{array}$ & $\begin{array}{l}14.0 \\
\quad(10.0-23.0)\end{array}$ & $\begin{array}{l}< \\
\quad 0.0- \\
01\end{array}$ \\
\hline Cost of hospitalization & 3367.5 & 3405.6 & 2637.3 & 2919.1 & $<$ \\
\hline (\$, median, IQR) & $\begin{array}{l}(1896.1 \\
\quad 10767.0)\end{array}$ & $\begin{array}{c}(2704.8 \\
10360.9)\end{array}$ & $\begin{array}{l}(1383.5, \\
10816.9)\end{array}$ & $\begin{array}{l}(1172.1 \\
15627.4)\end{array}$ & $\begin{array}{l}0.0- \\
01\end{array}$ \\
\hline 30 -day mortality $(n, \%)$ & $208(19.3)$ & $136(19.6)$ & $72(18.7)$ & $18(14.2)$ & 0.163 \\
\hline
\end{tabular}

$N A I$ neuraminidase inhibitor, $I C U$ intensive care unit. ${ }^{\dagger}$ Comparisons were made between patients with Flu-p and RSV-p. The bolded values are $p$-values $<0.05$, which represented significant differences between patients with Flu-p and RSV-p. and RSV-p, the risks were similar for all of the following: general risk for severe outcomes $(O R 1.506,95 \%$ CI 0.944 2.403, $p=0.086)$, invasive ventilation $(O R 0.805,95 \% C I$ $0.418-1.550, p=0.516)$, ICU admission $(O R 1.665,95 \% C I$
$0.900-3.080, p=0.104)$, and 30 -day mortality rate $(O R 1.389$, $95 \%$ CI 0.793-2.432, $p=0.251$ ).

A multivariate logistic regression analysis adjusting for age, sex, duration of illness onset to admission, comorbidities
Fig. 3 Forest plot of predictors for RSV-p. Age $\geq 50$ years, cerebrovascular disease, chronic kidney disease, solid malignant tumor, nasal congestion, respiratory rates $\geq 30$ beats $/ \mathrm{min}$, and blood albumin $<35 \mathrm{~g} / \mathrm{L}$ favored RSV-p; myalgia, sputum production, and lymphocytes $<$ $0.8 \times 10^{9} / \mathrm{L}$ favored Flu-p

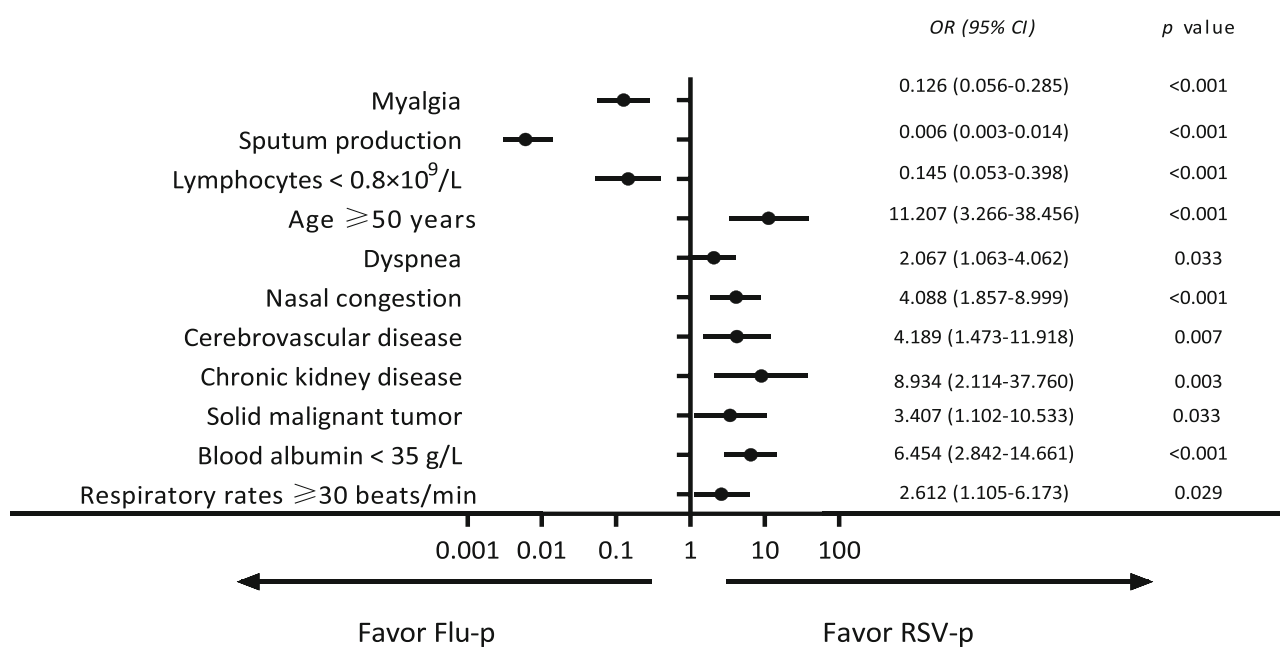


Table 3 Impact of viruses types on severe outcomes

\begin{tabular}{|c|c|c|c|c|c|}
\hline \multirow[t]{2}{*}{ Clinical outcomes } & \multirow{2}{*}{$\begin{array}{l}\text { Virus types } \\
\text { (r e fer e n c e: } \\
\text { RSV) }\end{array}$} & \multicolumn{2}{|c|}{ Univariate logistic analysis } & \multicolumn{2}{|c|}{ Multivariate logistic analysis } \\
\hline & & $O R(95 \% C I)$ & $P$-value & $*_{\mathrm{a} O R}(95 \% C I)$ & $P$-value \\
\hline \multirow[t]{2}{*}{ Severe outcomes } & Influenza A & $\begin{array}{l}1.931 \\
\quad(1.241-3.005)\end{array}$ & 0.004 & $\begin{array}{l}2.313 \\
\quad(1.377-3.885)\end{array}$ & 0.002 \\
\hline & Influenza B & $\begin{array}{l}1.506 \\
\quad(0.944-2.403)\end{array}$ & 0.086 & $\begin{array}{l}1.630 \\
\quad(0.958-2.741)\end{array}$ & 0.071 \\
\hline \multirow[t]{2}{*}{ Invasive ventilation } & Influenza A & $\begin{array}{l}2.384 \\
\quad(1.331-4.271)\end{array}$ & 0.003 & $\begin{array}{l}2.680 \\
\quad(1.393-5.154)\end{array}$ & 0.003 \\
\hline & Influenza B & $\begin{array}{l}0.805 \\
\quad(0.418-1.550)\end{array}$ & 0.516 & $\begin{array}{l}0.683 \\
\quad(0.333-1.400)\end{array}$ & 0.297 \\
\hline \multirow[t]{2}{*}{$\begin{array}{l}\text { Admittance to the } \\
\text { ICU }\end{array}$} & Influenza A & $\begin{array}{l}2.748 \\
\quad(1.537-4.913)\end{array}$ & 0.001 & $\begin{array}{l}2.067 \\
\quad(1.064-4.015)\end{array}$ & 0.032 \\
\hline & Influenza B & $\begin{array}{l}1.665 \\
\quad(0.900-3.080)\end{array}$ & 0.104 & $\begin{array}{l}1.994 \\
\quad(0.993-4.005)\end{array}$ & 0.052 \\
\hline \multirow[t]{2}{*}{ 30-day mortality } & Influenza A & $\begin{array}{l}1.479 \\
\quad(0.868-2.519)\end{array}$ & 0.150 & $\begin{array}{l}2.503 \\
\quad(1.229-5.101)\end{array}$ & 0.012 \\
\hline & Influenza B & $\begin{array}{l}1.389 \\
\quad(0.793-2.432)\end{array}$ & 0.251 & $\begin{array}{l}1.898 \\
\quad(0.937-3.846)\end{array}$ & 0.075 \\
\hline
\end{tabular}

OR odds ratio, $C I$ confidence interval, $a O R$ adjusted odds ratio. ${ }^{*}$ Adjusted for age, sex, duration from illness onset to admission, comorbidities (cardiovascular disease, cerebrovascular disease, diabetes mellitus, chronic pulmonary disease, asthma, chronic kidney disease, and solid malignant tumor), obesity, pregnancy, smoking history, systemic corticosteroid use, and coinfections (cardiovascular disease, cerebrovascular disease, diabetes mellitus, chronic obstructive pulmonary disease, asthma, chronic kidney disease, and solid malignant tumor), obesity, pregnancy, smoking history, administration of early NAI, systemic corticosteroid use during hospitalization, and coinfections indicated that, relative to RSV-p, FluA-p was associated with an increased risk for severe outcomes (adjusted OR $(a O R) 2.313,95 \%$ CI 1.377-3.885, $p=0.002$ ), including invasive ventilation ( $a O R 2.680,95 \% C I 1.393-5.154, p=$ 0.003 ), ICU admission ( $a O R 2.067,95 \%$ CI 1.064-4.015, $p=$ 0.032 ), and 30-day mortality ( $a O R 2.503$, 95\% CI 1.2295.101, $p=0.012$ ). Regarding FluB-p, compared with RSV$\mathrm{p}$, the risks were similar for all of the following: invasive ventilation ( $a O R \quad 0.683,95 \%$ CI $0.333-1.400, p=0.297$ ), ICU admission ( $a O R$ 1.994, 95\% CI 0.993-4.005, $p=$ 0.052), 30-day mortality ( $a O R 1.898,95 \%$ CI 0.937-3.846, $p=0.075)$, and total number of severe outcomes ( $a O R$ 1.630, 95\% CI 0.958-2.741, $p=0.071$ ) (Table 3 and Fig. 4).

Figure 5 shows that after adjusting for confounders, the 30day mortality rate for FluA-p patients was significantly higher than that of RSV-p patients (adjusted hazard ratio (aHR) $2.280,95 \%$ CI 1.203-4.312, $p=0.011)$. In contrast, the 30 day mortality rates for patients with FluB-p and RSV-p were similar (aHR 1.208, 95\% CI 0.650-2.246, $p=0.549)$.

\section{Risk factors for severe outcomes in RSV-p patients}

Compared to RSV-p patients without severe outcomes, RSV$\mathrm{p}$ patients with severe outcomes were older and were more likely to present with chronic pulmonary disease. The proportions of lymphocytes $<0.8 \times 10^{9} / \mathrm{L}$, hemoglobin $<100$ $\mathrm{g} / \mathrm{dL}$, blood urea nitrogen $>7 \mathrm{mmol} / \mathrm{L}$, and $\mathrm{PaO}_{2} / \mathrm{FiO}_{2}<$ $250 \mathrm{mmHg}$ at admission and the use of systemic corticosteroids during hospitalization were higher in RSV-p patients with severe outcomes than in RSV-p patients without severe outcomes (Supplementary Material 5).

To explore the risk factors for severe outcomes in RSV-p patients, the following variables were entered into a logistic regression model: age, chronic obstructive pulmonary disease, smoking history, confusion, leukocytes $>10 \times 10^{9} / \mathrm{L}$,

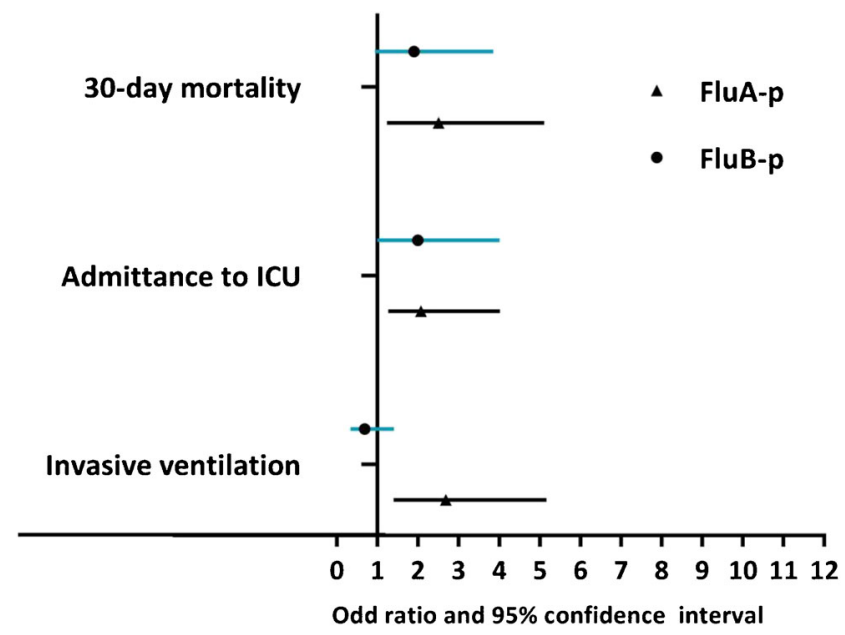

Fig. 4 Comparison of severity and outcomes by virus types (reference: RSV-p). Compared to RSV-p, FluA-p was associated with increased risks for invasive ventilation, ICU admission, and 30-day mortality; the risks for invasive ventilation, ICU admission, and 30-day mortality of FluB-p were not significantly different with RSV-p 


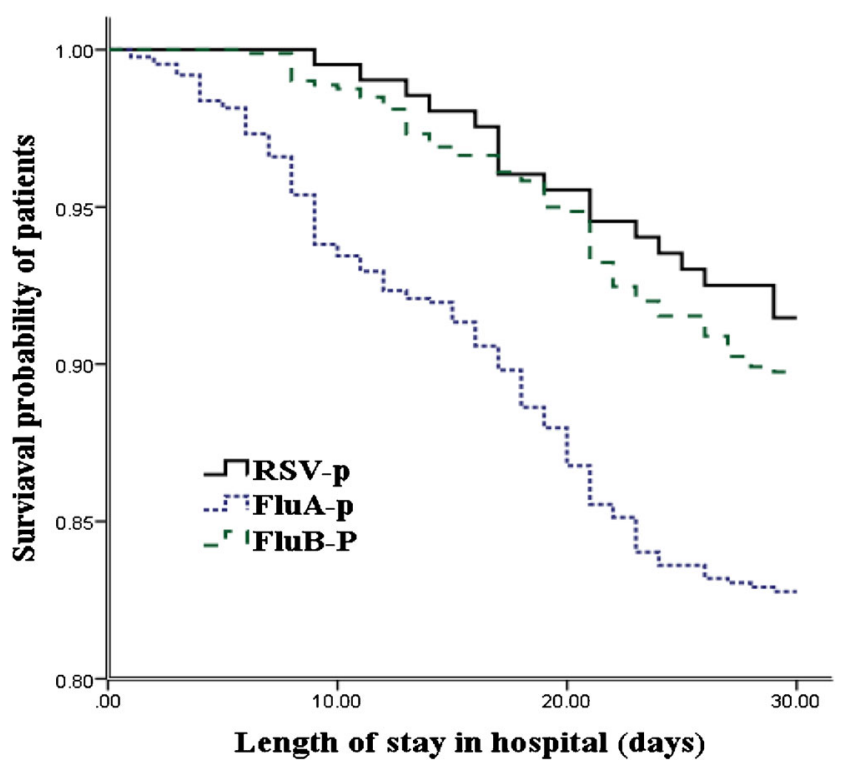

Fig. 5 Survival rate of patients hospitalized with FluA-p, FluB-p, and RSV-p (censored at 30 days after admission). After adjustment for confounders, the 30-day mortality of FluA-p patients was significantly higher than that of RSV-p patients, while the 30-day mortality of patients with FluB-p and RSV-p was similar

lymphocytes $<0.8 \times 10^{9} / \mathrm{L}$, hemoglobin $<100 \mathrm{~g} / \mathrm{dL}$, blood urea nitrogen $>7 \mathrm{mmol} / \mathrm{L}, \mathrm{PaO}_{2} / \mathrm{FiO}_{2}<250 \mathrm{mmHg}$, and systemic corticosteroid use. Results indicated that age (OR 1.084, 95\% CI 1.010-1.164, $p=0.026)$, chronic obstructive pulmonary disease $(O R 5.512,95 \%$ CI 1.721-17.652, $p=$ $0.004)$, confusion $(O R 8.293,95 \%$ CI 2.022-34.016, $p=$ $0.003)$, lymphocytes $<0.8 \times 10^{9} / \mathrm{L}(O R 6.011,95 \% C I$ 1.376-26.249, $p=0.017)$, and blood urea nitrogen $>7$ $\mathrm{mmol} / \mathrm{L}($ OR 3.588, 95\% CI 1.161-11.088, $p=0.026)$ at admission were statistically significant independent risk factors for severe outcomes in RSV-p patients (Table 4).

\section{Discussion}

In our large cohort study examining hospitalized patients with Flu-p and RSV-p, we found two important results: (i) although Flu-p and RSV-p exhibited similar epidemiology and clinical characteristics, some variables would be used potentially for

Table 4 Risk factors for severe outcomes in RSV-p patients

\begin{tabular}{lll}
\hline Risk factors & OR $(95 \%$ CI $)$ & $P$-value \\
\hline Age & $1.084(1.010-1.164)$ & 0.026 \\
COPD & $5.512(1.721-17.652)$ & 0.004 \\
Confusion & $8.293(2.022-34.016)$ & 0.003 \\
Lymphocytes $<0.8 \times 10^{9} / \mathrm{L}$ & $6.011(1.376-26.249)$ & 0.017 \\
BUN $>7 \mathrm{mmol} / \mathrm{L}$ & $3.588(1.161-11.088)$ & 0.026 \\
\hline
\end{tabular}

making differential diagnoses in the future and (ii) after adjusting for potential confounders, we found that RSV-p was comparable to FluB-p, but was less severe than FluA-p.

In our study, compared to Flu-p patients, patients with RSV-p were older and experienced more frequent chronic medical conditions and hypoproteinemia. Although cough was a common symptom, sputum production was relatively rare. Nasal congestion, dyspnea, and respiratory rates $\geq 30$ breaths/min were more common in RSV-p, whereas myalgia was more common in Flu-p patients. These findings are consistent with previous research. For example, Lee and colleagues [25] compared hospitalized patients with RSV and influenza and found that patients with RSV more often had systemic comorbidities, dry cough, wheezy breathing, and dyspnea. Data from a virological surveillance study of respiratory viruses in France showed that dyspnea was associated with an increased risk for RSV infection (OR 2.33, 95\% CI 1.73-3.12) and a decreased risk for influenza virus infection (OR 0.56, 95\% CI 0.46-0.70) [26]. The study by Casalegno and colleagues [27] suggested that myalgia and shortness of breath were useful for distinguishing influenza from other respiratory viruses. In Pedersen's study [28], myalgia was found to be an independent predictor of influenza for patients with acute respiratory infection. A study by Sundaram [10] examined the characteristics of RSV relative to other viral infections in adults aged 50 years and older and found that RSV was associated with advanced age (OR 1.50, 95\% CI 1.07-2.10), symptoms of nasal congestion (OR 2.15, 95\% $C I$ 1.32-3.52), and wheezing breath $(O R 1.81,95 \% C I$ 1.31-2.50).

In addition to the key findings discussed above, we found that lymphocytes $<0.8 \times 10^{9} / \mathrm{L}$ could discriminate between RSV-p and Flu-p infections. This finding appears to be novel and has not been previously reported. Lymphopenia was very common in severe influenza with an incidence rate of 30 $100 \%$ [29, 30] and was associated with reduced T lymphocytes in the peripheral blood [31]. Lymphopenia was also observed in RSV infection [32, 33]. However, previous studies suggested that decreased lymphocytes occurred only in critically ill children with RSV infections [33]. We suspect that lymphopenia is useful for differential diagnosis. Interestingly, we found that lymphopenia was associated with poor clinical outcomes. This finding is consistent with previous research on influenza or other viral pneumonias, in which lymphopenia was a predictor of increased mortality [34, 35]. A study by Vakil [36] likewise found that lymphopenia ( $O R$ $3.7,95 \%$ CI 1.7-8.2, $p=0.001$ ) was associated with 60-day mortality rate in hematologic malignancy patients with RSV infection. The mechanisms relating lymphopenia to RSV infection are not clear and require further investigation. A study with infants diagnosed with severe RSV bronchiolitis found that their plasma levels of soluble Fas ligand and caspase-1 were increased, which in turn caused the apoptosis of CD4+ 
helper lymphocytes and CD8+ cytotoxic lymphocytes [37]. That virus-induced $\mathrm{T}$ lymphocyte depletion and compromised cellular immunity delayed the clearance of the viruses. Another possible explanation is that lymphocytes were sequestered or recruited at respiratory tracts from the circulating blood [38]. The aggregation of lymphocytes in the lungs caused severe inflammation and damage to tissues. Although statistically significant, the absolute values of the differences in these clinical indicators between patients with Flu-p and RSV-p were only 10-30\%. If studied prospectively as a diagnostic indicator, these clinical indicators are likely to show low sensitivity and/or specificity for identification. We think the clinical features are helpful for differential diagnosis, especially in in resource-limited and primary hospitals, but not substitutes for etiological tests.

In our study, the 30-day mortality rate of RSV-p patients was $14.2 \%$, consistent with previous reports of a $2-20 \%$ mortality rate in hospitalized adult patients with RSV infection $[39,40]$. Few studies have directly compared the clinical features and severity of Flu-p and RSV-p. Some studies only investigated the outcomes (e.g., hospitalizations and deaths), but did not control for potential confounders (e.g., study settings, population type, treatments) [17, 41, 42]. In our study, after adjusting for confounders, we found that the severities of RSV-p and Flu-p were not the same. FluA-p was more severe than both RSV-p and FluB-p. Caini and colleagues [43] analyzed the severity of respiratory viral infections in Ecuador from 2009 to 2016 and found that after adjusting for age group, gender, year, type of surveillance scheme, and region, the risk of death for patients infected with A (H1N1) pdm09 was $1.73(1.38-2.17)$, which was higher than that for RSV $(0.75,0.57-0.98)$. Similarly, in a study by Minney-Smith et al. [44], patients with influenza B and RSV experienced milder outcomes, including ICU admission $(0.385$ (0.151$0.984), 0.389$ (0.162-0.936), respectively) and complications due to pneumonia $(0.269(0.130-0.557), 0.483(0.246-$ 0.951 ), respectively), relative to patients with influenza $\mathrm{A}$ (H1N1) pdm09, after controlling for age group, sex, and comorbidities. However, these researchers did not compare the severity of influenza B versus RSV. Our study did compare the outcomes of FluB-p and RSV-p and found the severity of the two infections to be similar. In both cases, the severity of pneumonia was influenced by the combination of host, viral, and environmental factors. Because we controlled for numerous potential confounders, our findings help to clarify the direct influence of virus type on clinical outcomes.

To our knowledge, this is the first cohort study that directly compared the clinical severity and outcomes of RSV-p to specific viral types of Flu-p. However, some limitations of our study should be noted. First, due to the retrospective nature of the study, potential selection bias could have impacted the results. Because attending physicians determined which patients received viral RNA tests, it is possible that more severe (or milder) patients were tested. Also, not all respiratory cases were eligible for swabbing, which is another factor that could induce some selection bias. Another limitation is that because of the retrospective design, the impact of vaccination on disease severity could not be evaluated. An additional limitation is that over $50 \%$ of the FluA-p patients and all of the RSV-p patients were not tested for viral subtypes. Further research is required to examine whether the clinical features vary across virus subtypes [45]. Finally, given that our study participants were immunocompetent adult hospitalized patients, our findings might not be readily generalize to other populations such as immunocompromised patients, children, and outpatients.

In summary, our results indicate that some clinical characteristics identified in our study would be helpful to discriminate RSV-p from Flu-p. What is more, the differences in disease severity between them highlight the importance of virus type tests in the clinical management of both severe influenza and RSV infections.

Supplementary Information The online version contains supplementary material available at https://doi.org/10.1007/s10096-021-04217-2.

Funding This study is founded by Beijing JST Research Funding (ZR201921).

Data availability All data generated or analyzed during this study are included in this published article/as supplementary information files.

\section{Declarations}

Ethics approval This study was conducted in accordance with the Declaration of Helsinki and was approved by the Ethics Committee of Beijing Jishuitan Hospital (No.201911-15). Given the retrospective nature of the study, the ethics committee determined that an informed consent was not necessary.

Informed consent Not applicable to this study.

Conflict of interest The authors declare no conflict of interest.

\section{References}

1. Fineberg HV (2014) Pandemic preparedness and response-lessons from the H1N1 influenza of 2009. N Engl J Med 370(14):13351342. https://doi.org/10.1056/NEJMra1208802

2. Abdel-Hady DM, Al Balushi RM, Al Abri BA, Al Abri SS, Al Kindi HS, Al-Jardani AK, Al Yaqubi FM, Al Abaidani IS (2018) Estimating the burden of influenza-associated hospitalization and deaths in Oman (2012-2015). Influenza Other Respir Viruses 12(1): 146-152. https://doi.org/10.1111/irv.12500

3. Jones RM, Xia Y (2018) Annual burden of occupationally-acquired influenza infections in hospitals and emergency departments in the United States. Risk Anal 38(3):442-453. https://doi.org/10.1111/ risa. 12854 
4. Ly S, Arashiro T, Ieng V, Tsuyuoka R, Parry A, Horwood P, Heng S, Hamid S, Vandemaele K, Chin S, Sar B, Arima Y (2017) Establishing seasonal and alert influenza thresholds in Cambodia using the WHO method: implications for effective utilization of influenza surveillance in the tropics and subtropics. Western Pac Surveill Response J 8(1):22-32. https://doi.org/10.5365/WPSAR. 2017.8.1.002

5. Stein RT, Bont LJ, Zar H, Polack FP, Park C, Claxton A, Borok G, Butylkova Y, Wegzyn C (2017) Respiratory syncytial virus hospitalization and mortality: systematic review and meta-analysis. Pediatr Pulmonol 52(4):556-569. https://doi.org/10.1002/ppul. 23570

6. Kodama F, Nace DA, Jump RLP (2017) Respiratory syncytial virus and other noninfluenza respiratory viruses in older adults. Infect Dis Clin N Am 31(4):767-790. https://doi.org/10.1016/j.idc.2017.07. 006

7. Malosh RE, Martin ET, Callear AP, Petrie JG, Lauring AS, Lamerato L, Fry AM, Ferdinands J, Flannery B, Monto AS (2017) Respiratory syncytial virus hospitalization in middle-aged and older adults. J Clin Virol 96:37-43. https://doi.org/10.1016/j. jev.2017.09.001

8. Falsey AR, Hennessey PA, Formica MA, Cox C, Walsh EE (2005) Respiratory syncytial virus infection in elderly and high-risk adults. N Engl J Med 352(17):1749-1759. https://doi.org/10.1056/ NEJMoa043951

9. Matias G, Taylor R, Haguinet F, Schuck-Paim C, Lustig R, Shinde V (2014) Estimates of mortality attributable to influenza and RSV in the United States during 1997-2009 by influenza type or subtype, age, cause of death, and risk status. Influenza Other Respir Viruses 8(5):507-515. https://doi.org/10.1111/irv. 12258

10. Sundaram ME, Meece JK, Sifakis F, Gasser RA Jr, Belongia EA (2014) Medically attended respiratory syncytial virus infections in adults aged $\geq 50$ years: clinical characteristics and outcomes. Clin Infect Dis 58(3):342-349. https://doi.org/10.1093/cid/cit767

11. Griffiths C, Drews SJ, Marchant DJ (2017) Respiratory syncytial virus: infection, detection, and new options for prevention and treatment. Clin Microbiol Rev 30(1):277-319. https://doi.org/10.1128/ CMR.00010-16

12. Jain S, Self WH, Wunderink RG, Fakhran S, Balk R, Bramley AM, Reed C, Grijalva CG, Anderson EJ, Courtney DM, Chappell JD, Qi C, Hart EM, Carroll F, Trabue C, Donnelly HK, Williams DJ, Zhu Y, Arnold SR, Ampofo K, Waterer GW, Levine M, Lindstrom S, Winchell JM, Katz JM, Erdman D, Schneider E, Hicks LA, McCullers JA, Pavia AT, Edwards KM, Finelli L, Team CES (2015) Community-acquired pneumonia requiring hospitalization among U.S. adults. N Engl J Med 373(5):415-427. https://doi. org/10.1056/NEJMoa1500245

13. Nam HH, Ison MG (2019) Respiratory syncytial virus infection in adults. BMJ 366:15021. https://doi.org/10.1136/bmj.15021

14. Ackerson B, Tseng HF, Sy LS, Solano Z, Slezak J, Luo Y, Fischetti CA, Shinde V (2019) Severe morbidity and mortality associated with respiratory syncytial virus versus influenza infection in hospitalized older adults. Clin Infect Dis 69(2):197-203. https://doi.org/ 10.1093/cid/ciy991

15. Kestler M, Munoz P, Mateos M, Adrados D, Bouza E (2018) Respiratory syncytial virus burden among adults during flu season: an underestimated pathology. J Hosp Infect 100(4):463-468. https://doi.org/10.1016/j.jhin.2018.03.034

16. Malley R, DeVincenzo J, Ramilo O, Dennehy PH, Meissner HC, Gruber WC, Sanchez PJ, Jafri H, Balsley J, Carlin D, Buckingham S, Vernacchio L, Ambrosino DM (1998) Reduction of respiratory syncytial virus (RSV) in tracheal aspirates in intubated infants by use of humanized monoclonal antibody to RSV F protein. J Infect Dis 178(6):1555-1561. https://doi.org/10.1086/314523

17. Jansen AG, Sanders EA, Hoes AW, van Loon AM, Hak E (2007) Influenza- and respiratory syncytial virus-associated mortality and hospitalisations. Eur Respir J 30(6):1158-1166. https://doi.org/10. 1183/09031936.00034407

18. Bednarska K, Hallmann-Szelinska E, Kondratiuk K, Brydak LB (2015) Evaluation of the activity of influenza and influenza-like viruses in the epidemic season 2013/2014. Adv Exp Med Biol 857:1-7. https://doi.org/10.1007/5584_2015_116

19. Panozzo CA, Fowlkes AL, Anderson LJ (2007) Variation in timing of respiratory syncytial virus outbreaks: lessons from national surveillance. Pediatr Infect Dis J 26(11 Suppl):S41-S45. https://doi. org/10.1097/INF.0b013e318157da82

20. Chen L, Zhou F, Li H, Xing X, Han X, Wang Y, Zhang C, Suo L, Wang J, Yu G, Wang G, Yao X, Yu H, Wang L, Liu M, Xue C, Liu B, Zhu X, Li Y, Xiao Y, Cui X, Li L, Uyeki TM, Wang C, Cao B, network CA-C (2018) Disease characteristics and management of hospitalised adolescents and adults with community-acquired pneumonia in China: a retrospective multicentre survey. BMJ Open 8 (2):e018709. https://doi.org/10.1136/bmjopen-2017-018709.

21. Chatzis O, Darbre S, Pasquier J, Meylan P, Manuel O, Aubert JD, Beck-Popovic M, Masouridi-Levrat S, Ansari M, Kaiser L, PosfayBarbe KM, Asner SA (2018) Burden of severe RSV disease among immunocompromised children and adults: a 10 year retrospective study. BMC Infect Dis 18(1):111. https://doi.org/10.1186/s12879018-3002-3

22. Kossyvakis A, Mentis AA, Tryfinopoulou K, Pogka V, Kalliaropoulos A, Antalis E, Lytras T, Meijer A, Tsiodras S, Karakitsos P, Mentis AF (2017) Antiviral susceptibility profile of influenza A viruses; keep an eye on immunocompromised patients under prolonged treatment. Eur J Clin Microbiol Infect Dis 36(2): 361-371. https://doi.org/10.1007/s10096-016-2809-3

23. Muthuri SG, Venkatesan S, Myles PR, Leonardi-Bee J, Al Khuwaitir TS, Al Mamun A, Anovadiya AP, Azziz-Baumgartner E, Baez C, Bassetti M, Beovic B, Bertisch B, Bonmarin I, Booy R, Borja-Aburto VH, Burgmann H, Cao B, Carratala J, Denholm JT, Dominguez SR, Duarte PA, Dubnov-Raz G, Echavarria M, Fanella S, Gao Z, Gerardin P, Giannella M, Gubbels S, Herberg J, Iglesias AL, Hoger PH, Hu X, Islam QT, Jimenez MF, Kandeel A, Keijzers G, Khalili H, Knight M, Kudo K, Kusznierz G, Kuzman I, Kwan AM, Amine IL, Langenegger E, Lankarani KB, Leo YS, Linko R, Liu P, Madanat F, Mayo-Montero E, McGeer A, Memish Z, Metan G, Mickiene A, Mikic D, Mohn KG, Moradi A, Nymadawa P, Oliva ME, Ozkan M, Parekh D, Paul M, Polack FP, Rath BA, Rodriguez AH, Sarrouf EB, Seale AC, Sertogullarindan B, Siqueira MM, Skret-Magierlo J, Stephan F, Talarek E, Tang JW, To KK, Torres A, Torun SH, Tran D, Uyeki TM, Van Zwol A, Vaudry W, Vidmar T, Yokota RT, Zarogoulidis P, Investigators PC, Nguyen-Van-Tam JS (2014) Effectiveness of neuraminidase inhibitors in reducing mortality in patients admitted to hospital with influenza A H1N1pdm09 virus infection: a meta-analysis of individual participant data. Lancet Respir Med 2(5):395-404. https:// doi.org/10.1016/S2213-2600(14)70041-4

24. Ku YH, Chan KS, Yang CC, Tan CK, Chuang YC, Yu WL (2017) Higher mortality of severe influenza patients with probable aspergillosis than those with and without other coinfections. J Formos Med Assoc 116(9):660-670. https://doi.org/10.1016/j.jfma.2017. 06.002

25. Lee N, Lui GC, Wong KT, Li TC, Tse EC, Chan JY, Yu J, Wong SS, Choi KW, Wong RY, Ngai KL, Hui DS, Chan PK (2013) High morbidity and mortality in adults hospitalized for respiratory syncytial virus infections. Clin Infect Dis 57(8):1069-1077. https://doi. org $/ 10.1093 / \mathrm{cid} / \mathrm{cit} 471$

26. Loubet P, Lenzi N, Valette M, Foulongne V, Krivine A, Houhou N, Lagathu G, Rogez S, Alain S, Duval X, Galtier F, Postil D, Tattevin P, Vanhems P, Carrat F, Lina B, Launay O, Group FS (2017) Clinical characteristics and outcome of respiratory syncytial virus infection among adults hospitalized with influenza-like illness in 
France. Clin Microbiol Infect 23(4):253-259. https://doi.org/10. 1016/j.cmi.2016.11.014

27. Casalegno JS, Eibach D, Valette M, Enouf V, Daviaud I, Behillil S, Vabret A, Soulary JC, Benchaib M, Cohen JM, van der Werf S, Mosnier A, Lina B (2017) Performance of influenza case definitions for influenza community surveillance: based on the French influenza surveillance network GROG, 2009-2014. Euro Surveill 22(14):30504. https://doi.org/10.2807/1560-7917.ES.2017.22.14. 30504

28. Pedersen CJ, Quinn JV, Rogan DT, Yang S (2019) Factors associated with influenza in an emergency department setting. J Emerg Med 56(5):478-483. https://doi.org/10.1016/j.jemermed.2018.12. 012

29. Lalueza A, Folgueira D, Diaz-Pedroche C, Hernandez-Jimenez P, Ayuso B, Castillo C, Laureiro J, Trujillo H, Torres M, Lumbreras C (2019) Severe lymphopenia in hospitalized patients with influenza virus infection as a marker of a poor outcome. Infect Dis (Lond) 51(7):543-546. https://doi.org/10.1080/23744235.2019.1598572

30. Bellelli V, d'Ettorre G, Celani L, Borrazzo C, Ceccarelli G, Venditti M (2019) Clinical significance of lymphocytopenia in patients hospitalized with pneumonia caused by influenza virus. Crit Care 23(1):330. https://doi.org/10.1186/s13054-019-2608-1

31. Fox A, Le NM, Horby P, van Doorn HR, Nguyen VT, Nguyen HH, Nguyen TC, Vu DP, Nguyen MH, Diep NT, Bich VT, Huong HT, Taylor WR, Farrar J, Wertheim H, Nguyen VK (2012) Severe pandemic H1N1 2009 infection is associated with transient NK and T deficiency and aberrant CD8 responses. PLoS One 7(2):e31535. https://doi.org/10.1371/journal.pone.0031535

32. Russell CD, Unger SA, Walton M, Schwarze J (2017) The human immune response to respiratory syncytial virus infection. Clin Microbiol Rev 30(2):481-502. https://doi.org/10.1128/CMR. 00090-16

33. O'Donnell DR, Carrington D (2002) Peripheral blood lymphopenia and neutrophilia in children with severe respiratory syncytial virus disease. Pediatr Pulmonol 34(2):128-130. https://doi.org/10.1002/ ppul.10140

34. Shi SJ, Li H, Liu M, Liu YM, Zhou F, Liu B, Qu JX, Cao B (2017) Mortality prediction to hospitalized patients with influenza pneumonia: $\mathrm{PO} 2 / \mathrm{FiO} 2$ combined lymphocyte count is the answer. Clin Respir J 11(3):352-360. https://doi.org/10.1111/crj.12346

35. Guo L, Wei D, Zhang X, Wu Y, Li Q, Zhou M, Qu J (2019) Clinical features predicting mortality risk in patients with viral pneumonia: the MuLBSTA score. Front Microbiol 10:2752. https://doi.org/10. 3389/fmicb.2019.02752

36. Vakil E, Sheshadri A, Faiz SA, Shah DP, Zhu Y, Li L, Kmeid J, Azzi J, Balagani A, Bashoura L, Ariza-Heredia E, Chemaly RF (2018) Risk factors for mortality after respiratory syncytial virus lower respiratory tract infection in adults with hematologic malignancies. Transpl Infect Dis 20(6):e12994. https://doi.org/10.1111/ tid. 12994
37. Roe MF, Bloxham DM, White DK, Ross-Russell RI, Tasker RT, O'Donnell DR (2004) Lymphocyte apoptosis in acute respiratory syncytial virus bronchiolitis. Clin Exp Immunol 137(1):139-145. https://doi.org/10.1111/j.1365-2249.2004.02512.x

38. Smith PK, Wang SZ, Dowling KD, Forsyth KD (2001) Leucocyte populations in respiratory syncytial virus-induced bronchiolitis. J Paediatr Child Health 37(2):146-151. https://doi.org/10.1046/j. 1440-1754.2001.00618.x

39. Kim HJ, Choi SM, Lee J, Park YS, Lee CH, Yim JJ, Yoo CG, Kim YW, Han SK, Lee SM (2018) Respiratory virus of severe pneumonia in South Korea: prevalence and clinical implications. PLoS One 13(6):e0198902. https://doi.org/10.1371/journal.pone.0198902

40. Gamino-Arroyo AE, Moreno-Espinosa S, Llamosas-Gallardo B, Ortiz-Hernandez AA, Guerrero ML, Galindo-Fraga A, GalanHerrera JF, Prado-Galbarro FJ, Beigel JH, Ruiz-Palacios GM, Noyola DE, Mexico Emerging Infectious Diseases Clinical Research N (2017) Epidemiology and clinical characteristics of respiratory syncytial virus infections among children and adults in Mexico. Influenza Other Respir Viruses 11(1):48-56. https://doi. org/10.1111/irv.12414

41. Katsurada N, Suzuki M, Aoshima M, Yaegashi M, Ishifuji T, Asoh $\mathrm{N}$, Hamashige N, Abe M, Ariyoshi K, Morimoto K, Adult Pneumonia Study G-J (2017) The impact of virus infections on pneumonia mortality is complex in adults: a prospective multicentre observational study. BMC Infect Dis 17(1):755. https://doi.org/10. 1186/s12879-017-2858-y

42. Mullooly JP, Bridges CB, Thompson WW, Chen J, Weintraub E, Jackson LA, Black S, Shay DK, Vaccine Safety Datalink Adult Working G (2007) Influenza- and RSV-associated hospitalizations among adults. Vaccine 25(5):846-855. https://doi.org/10.1016/j. vaccine.2006.09.041

43. Caini S, de Mora D, Olmedo M, Portugal D, Becerra MA, Mejia M, Pacurucu MC, Ojeda J, Bonaccorsi G, Lorini C, Paget J, Bruno A (2019) The epidemiology and severity of respiratory viral infections in a tropical country: Ecuador, 2009-2016. J Infect Public Health 12(3):357-363. https://doi.org/10.1016/j.jiph.2018.12.003

44. Minney-Smith CA, Selvey LA, Levy A, Smith DW (2019) Postpandemic influenza A/H1N1pdm09 is associated with more severe outcomes than $\mathrm{A} / \mathrm{H} 3 \mathrm{~N} 2$ and other respiratory viruses in adult hospitalisations. Epidemiol Infect 147:e310. https://doi.org/10. 1017/S095026881900195X

45. Ishiguro $\mathrm{T}$, Takayanagi N, Kanauchi T, Uozumi R, Kawate E, Takaku Y, Kagiyama N, Shimizu Y, Hoshi T, Morita S, Sugita Y (2016) Clinical and radiographic comparison of influenza virusassociated pneumonia among three viral subtypes. Intern Med 55(7):731-737. https://doi.org/10.2169/internalmedicine.55.5227

Publisher's note Springer Nature remains neutral with regard to jurisdictional claims in published maps and institutional affiliations. 